\title{
Context Specific Descriptors for Tracking Deforming Tissue
}

\author{
Peter Mountney and Guang-Zhong Yang
}

The Hamlyn Centre for Robotic Surgery

Imperial College London

London, SW7 2AZ

UK

Preliminary results presented at MICCAI 2008, New York

Address for correspondence

Peter Mountney

The Hamlyn Centre for Robotic Surgery

Telephone: +44 (0) 2075948441

Imperial College

Fax: +44 (0) 2075818024

London SW7 2AZ, UK

Email: peter.mountney05@imperial.ac.uk 


\title{
Context Specific Descriptors for Tracking Deforming Tissue
}

\begin{abstract}
.
In minimally invasive surgery, deployment of motion compensation, dynamic active constraints and adaptive intra-operative guidance require accurate estimation of deforming tissue in 3D. To this end, the use of vision-based techniques is advantageous in that it does not require the integration of additional hardware to the existing surgical settings. Deformation can be recovered by tracking features on the surface of the tissue. Existing methods are mostly based on ad hoc machine vision techniques that have generally been developed for rigid scenes or use pre-determined models with parameters fine tuned to specific surgical settings. In this work, we propose a novel tracking technique based on a context specific feature descriptor. The descriptor can adapt to its surroundings and identify the most discriminate information for tracking. The feature descriptor is represented as a decision tree and the tracking process is formulated as a classification problem for which log likelihood ratios are used to improve classifier training. A recursive tracking algorithm is used to obtain examples of tissue deformation used to train the classifier. Additional training data is generated by geometric and appearance modeling. Experimental results have shown that the proposed context specific descriptor is robust to drift, occlusion, and changes in orientation and scale. The performance of the algorithm is compared with existing tracking algorithms and validated with both simulated and in vivo datasets.
\end{abstract}




\section{Introduction}

Recent advances in Minimally Invasive Surgery (MIS) have led to reduced patient trauma and hospitalization, making it popular with both patients and healthcare providers. For surgeons, MIS requires extensive manual dexterity and hand-eye coordination to deal with the loss of 3D vision, limited field-of-view and misalignment of the visuomotor axes. The introduction of robotic assisted MIS provides surgeons with improved 3D visualisation, enhanced dexterity, motion scaling and tremor cancellation. Robotic devices offer a potential platform for delivering technologies that can extend the current functions of MIS. These include integrating patient-specific, pre- and intra-operative data for image guided surgical navigation and intervention, adaptive motion compensation, dynamic view expansion and imposing dynamic active constraints or virtual fixtures. For these technologies to be successful, particularly for cardiovascular and gastrointestinal surgeries where large scale tissue deformation is common, an important prerequisite is accurate $3 \mathrm{D}$ estimation and tracking of dynamic tissue deformation in vivo.

In general, tissue deformation can be estimated using techniques such as intra-operative ultrasound, structured light (Fuchs et al., 1998) (Albitar et al., 2007), eye tracking (Mylonas et al., 2005) and time-of-flight cameras (Höller et al., 2008). Most of these approaches, however, require the introduction of additional hardware into the current operating environment. One key component of any MIS procedure is the endoscope camera and recent work has focussed on using this to perform tissue tracking and 3D surface/deformation recovery. For example, shape form shading has been used in (Quartucci Forster, 2000) (Okatani and Deguchi, 1997) (Deligianni et al., 2003) to recover the 3D structure of tissue from single monocular images by assuming the surface is perfectly Lambertian, the light source is at the optical centre and the surface material is uniform. The introduction of stereo laparoscopes has enabled the application of computational stereo with the use of priors. However, in order to recover the dynamic motion of the tissue, it is necessary to track the imaging sequences temporally. To this end, feature tracking techniques have been used (Ortmaier et al., 2005) (Stoyanov et al., 2005) (Mountney et al., 2007). In these approaches, features or salient regions are detected on the tissue surface. The features can be matched between stereo pairs to recover 3D structure or tracked temporally to recover tissue deformation. Multiple feature tracking is well-suited to deforming objects as it does not require the use of global models or prior information. Furthermore, it is capable of dealing with partial scene occlusion and specular highlights. 
Two major issues identified in the current vision based techniques are tracked feature density and feature persistency. Feature density refers to the number of features that can be tracked and it determines the accuracy of the 3D surface reconstruction. Feature density is dictated by the surface of the tissue in terms of homogeneity, texture and salient structural landmarks as illustrated in Figure 1. Structured light (Fuchs et al., 1998) can be used to constrain this problem by projecting patterns onto the surface of the organs, although this alters the surgeon's view unless an invisible frequency band is used. In (Devernay et al., 2001), correlation based dense stereo matching is used to create a 3D model of the heart. Local approaches to dense reconstruction such as this can be erroneous if the surface lacks texture or contains specular highlights. In (Richa et al., 2008) and (Lau et al., 2004), spline surfaces are fitted to data to estimate the 3D structure of textureless regions.

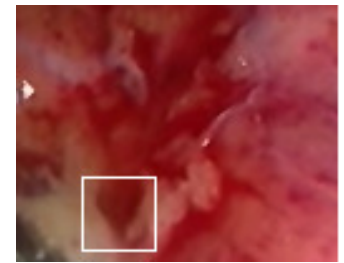

(a)

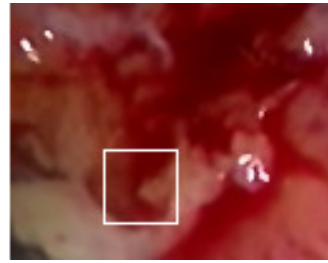

(b)

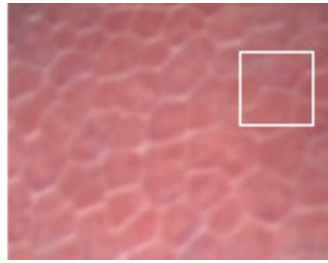

(c)

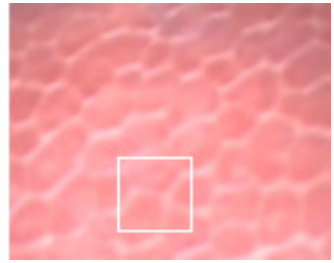

(d)

Fig. 1. Challenges associated with feature tracking in MIS. (a) and (b) illustrate the affect of deformation on the cardiac surface. The tracked feature, indicated with the white box, significantly changes shape. (c) and (d) demonstrate the repetitive pattern on the liver which makes distinguishing a feature from its surroundings difficult. These images are examples of the variety of the visual appearance of organs.

Feature persistency is related to the duration that a feature is reliably tracked and it influences how well tissue deformation can be modelled. Temporal feature tracking in MIS is challenging because it can be affected by occlusion (caused by specular highlights, tools, blood and smoke resulting from the use of diathermy) and changes in visual appearance (scale, orientation, illumination and nonlinear tissue deformation) as shown in Figure 1. Thus far, a number of approaches have been used to address the persistency problem in MIS. In (Ginhoux et al., 2004; Sauvee et al., 2006) fiducial markers have been added to the surface of tissue and in (Ott et al., 2008), the surface of the liver is burnt to create a trackable feature. Passive approaches based only on vision have been used in (Gröger et al., 2002; Koppel et al., 2007; Masson et al., 2009; Noce et al., 2007; Noce et al., 2006; Stoyanov D. et al., 2004; Wengert et al., 2006) to track the organ surfaces. However, these methods impose strong geometric constraints and generally do not explicitly model non-linear tissue deformation. As shown in (Masson et al., 2009), they may require empirical parameterisation for performance optimisation for a given scene or organ. Approaches such as (Koppel et al., 2007; Wengert et 
al., 2006) use descriptors that are invariant to image transformations and distortions, they have been shown to work well in rigid environments but can fail if the transformation is not modelled properly. In addition, ad hoc methods, can be susceptible to drift, non-continuous tracking and occlusion. Recently, a hybrid approach has been proposed (Richa et al., 2010) to deal with the weaknesses of individual tracking methods.

The ability to successfully track a feature is governed by how distinguishable it is from its surroundings. This is largely determined by the representation of the feature, which consists of two elements: 1) what information is represented and 2) how the information is represented. For the former, the information can include colour, edges, lines, corners, scale, orientation, intensity, texture or gradient. This information should contain sufficient variance to enable features to be separated from their surroundings whilst allowing them to undergo changes in appearance. For the latter, information may be represented as probability density histograms, histograms of gradients, templates, points, contours or active appearance models. The choice of what information to represent and how it is represented is context specific. Methods such as mean-shift (Comaniciu et al., 2003), Lucas Kanade (LK) (Lucas and Kanade, 1981) and Scale Invariant Feature Transform (SIFT) (Lowe, 2004) make prior, ad hoc decisions about what information to represent and how to represent it.

An alternative approach is to make use of contextual information and learn what makes a feature distinguishable from its surroundings. This learning approach has been used in hand writing recognition (Amit and Geman, 1997), object detection (Lepetit et al., 2005) and corner detection (Rosten and Drummond, 2006). Example datasets are created offline and used as training data to learn the most discriminative information. Offline learning requires prior knowledge of the data which is not available during MIS. In this work, we propose learning context specific information online to facilitate feature tracking.

The main focus of this paper is to describe in detail a method that can learn a context specific descriptor so as to improve feature density and persistency. To this end, feature tracking is formulated as a classification problem, for which we have proposed practical solutions for training the classifier with unlabeled data. This enables the algorithm to learn and subsequently use the best information for discriminating a feature from its surroundings. The approach does not require assumptions about the type of image transformations, enabling it to deal with nonlinear tissue deformation. The work presented here extends our previous work (Mountney and Yang, 2008) to make the method robust to scale change and rotation. In addition, the tracker is shown to be resilient to smoke artefacts from diathermy, which is used extensively in MIS for dissection and coagulation. A recent study (Spearman, 2007) showed 
$43 \%$ of surgeons take explicit action to clear away diathermy induced smoke to improve vision so the proposed method is practically important. The performance of the algorithm is compared to other existing tracking methods and validated with simulated, as well as in vivo MIS datasets. The strength of the algorithm in dealing with drift, occlusion, scale change, orientation change and smoke as well as tissue deformation is demonstrated.

\section{Methods}

In this section, we will describe how a feature is tracked and how context specific information is extracted and used to learn a feature descriptor. The proposed framework as illustrated in Figure 2, which consists of feature tracking and learning the context specific descriptor.

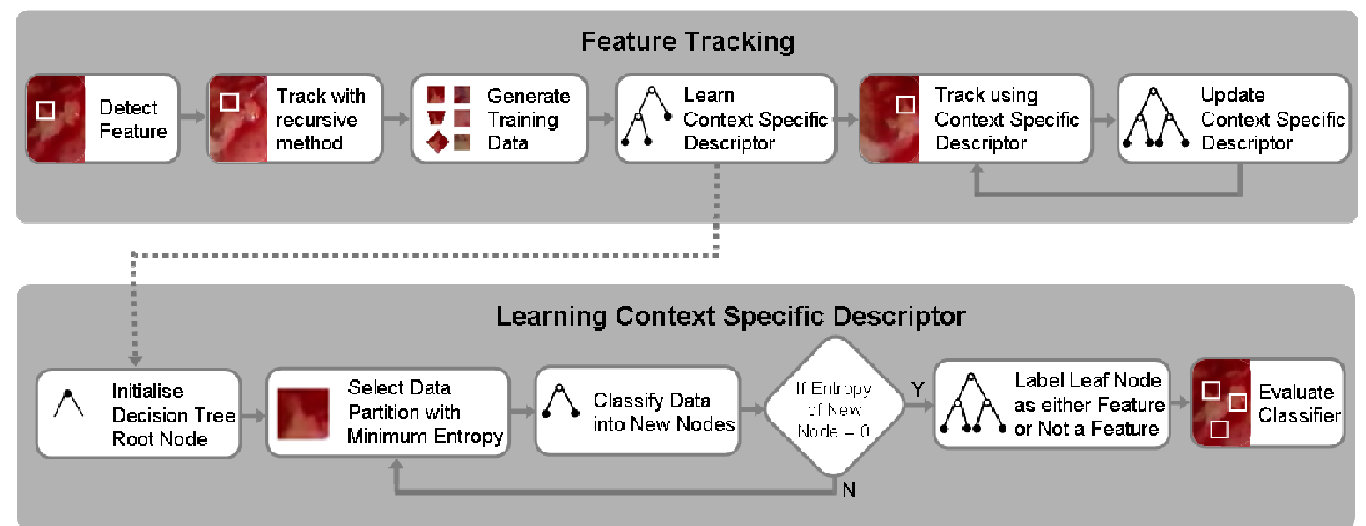

Fig. 2. A schematic diagram of the main processing steps involved in the proposed framework. The diagram outlines the fundamental steps in feature tracking and learning the context specific descriptor.

\subsection{Feature Tracking}

The framework employs two components to track a feature; a recursive method and a context specific descriptor. The feature is initially tracked for as short as $0.5 \mathrm{~s}$ using a recursive method. This enables a set of training data to be extracted from the video. The training data is then used to learn the context specific descriptor. Learning the descriptor and recursive tracking can be done in parallel. Once learnt, the descriptor is used to track the feature and is adaptively updated. The following section will describe each component of the feature tracking framework.

\subsubsection{Feature Detection and Recursive Tracking}

Features for tacking are extracted in the first video frame using Difference of Gaussian (DoG) (Lowe, 2004) and Shi and Tomasi (Shi and Tomasi, 1994) detectors. The detectors are combined to ensure features are extracted on a variety of tissue surfaces such as those 
illustrated in Figure 1. Features close to specular highlights are discarded. In this paper, specular highlights are identified by thresholding in Hue, Saturation and Value (HSV) space.

A feature is initially tracked with a recursive tracker to generate a training set with which to learn the context specific descriptor. This generates training data that contains examples of the feature deforming over time. For this purpose, any tracking method may be employed; however, the tracker must work with no prior knowledge of the feature or the image transformation it involved. In this study, a Lucas Kanade (LK) (Lucas and Kanade, 1981) tracker is used. Recursive techniques such as LK are well-suited to tracking deformation because they converge on the best solution. The major drawbacks of LK in MIS are cumulative error propagation and its sensitivity to illumination changes and occlusion. These drawbacks are minimised by limiting the duration that the feature is tracked using LK. In this framework, it is only used for a short period to generate a training dataset.

\subsubsection{Generate Training Data}

The set of training data $S$ is used to learn a feature descriptor. The set $S$ contains image patches. Each image patch has a label; feature or not a feature. Image patches with the label feature are examples of how the feature appears under image transformation (e.g. scale, rotation, deformation etc.). Image patches with the label not a feature are image patches that do not correspond to the feature. Figure 3 contains example image patches. In practice, generating training data online is challenging as there is no prior information about tissue deformation and manual labelling is not practical. To solve this problem, we propose creating a set of automatically labelled data using the results from recursive tracking. The training set is augmented with synthetically generated data. This makes it more robust to occlusion and rapid changes in appearance during MIS.

\section{Automatically Labelling Training Data}

During initial recursive tracking, an image patch centred on the position of the tracked feature is extracted. The patch is labelled as feature and added to the training set $S$. An example of training data labelled using the recursive tracker is shown in Figure 3. This approach creates a training set that captures the deformation of the feature without having to explicitly model tissue deformation. The descriptor will therefore be trained on context specific deformation. It is particularly suited to the heart, liver and other organs affected by periodic motion.

The set of image patches with label not a feature may be generated by taking an image patch centred at every position in the image that is not the position of the feature. This will create a 
large dataset, which is computationally prohibitive and can contain redundant data. Two approaches are used for extracting context specific training data; randomly selecting image patches and identifying visually similar patches. Randomly selecting patches from the video data ensures a variety of patches will be included in the training set $S$ with the label not $a$ feature. Examples of these patches are shown in Figure 3. Patches in the image which are visually similar to the feature are identified using normalised cross correlation template matching. These image patches are labelled and added to $S$. The context specific descriptor will be trained to distinguish the feature from these visually similar patches to reduce tracking errors.

\section{Generating synthetic training data}

The tracker's robustness to large image transformation can be improved by incorporating synthetically generated data. Generating synthetic data requires a prior model of the expected image transformation. In this study, two models are used; a geometric and an appearance based model. Geometric methods model the changes in geometry that cause the feature's appearance to alter in the image. Appearance based methods use statistical measures to model change in appearance. As an example, an appearance method is proposed in this paper for modelling the effect of diathermy-induced smoke.

The geometric method models three projective image transformations; scale, rotation and perspective. The warp models are individually applied to the automatically labelled data in $S$. This generates additional synthetic data depicted in Figure 3. The parameters of the model control the extent of the warping. To control the size of the training dataset the variance in parameter values has limited range and coarseness. Rotational transformations are applied in this study from $-45^{\circ}$ to $45^{\circ}$ at $2^{\circ}$ intervals. The scale transformations are applied from a factor of 0.8 to 3 to enable the tracker to handle similar transformation as (Lowe, 2004).
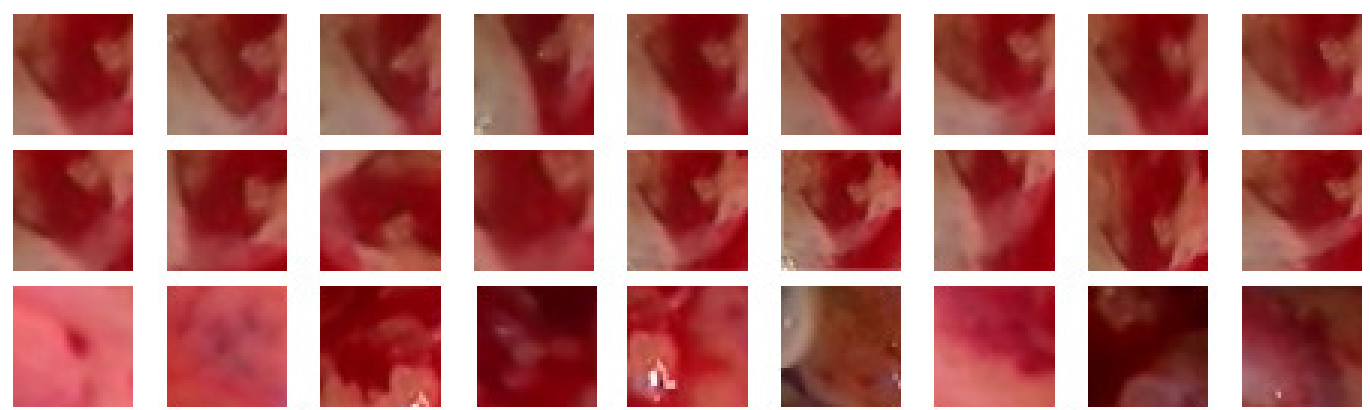

Fig. 3. Training datasets used for feature tracking. Top - examples of feature image patches of deformation acquired by recursive tracking. Middle - synthetically generated feature image patches for rotation, scale and perspective. Bottom - examples of not a feature image patches. 
The appearance based method models the effect of translucent diathermy-induced smoke. The translucent smoke causes tracking to fail because it can significantly change the appearance of the feature. Accurate 3D geometric modelling would require the detection of the smoke source, estimation of the smoke density and a 3D model of the peritoneal cavity with knowledge of the input and output of carbon dioxide. Appearance based methods enable surgical smoke to be effectively modelled with fewer parameters.

The proposed model has three variables; colour, density and a 2D spatial distribution of the smoke. These variables are combined in Equation 1 with the original pixel values from the image, in order to synthesise the visual appearance of smoke:

$$
\begin{aligned}
& P_{r^{\prime}}=d^{*} P_{r}+(1-d) C_{r} \\
& P_{g^{\prime}}=d^{*} P_{g}+(1-d) C_{g} \\
& P_{b^{\prime}}=d^{*} P_{b}+(1-d) C_{b}
\end{aligned}
$$

where $P_{r}$ is the original red component of the pixel, $C_{r}$ is the colour of the smoke (a random variable of Gaussian distribution with mean of 0.6 and standard deviation of 0.1$), d$ is a random variable representing smoke density, and $P_{r^{\prime}}$ is the transformed red component of the pixel. In this study, three different smoke density distributions are used with means 0.15 , 0.25 , and 0.4 with standard deviations of 0.05 . These values are illustrated in Figure 4 and are chosen because they represent translucent smoke. Values below 0.1 have little effect on visual appearance while those above 0.5 may result in occlusion. A Gaussian filter is applied to the resulting images to create smooth spatial distribution. The smoke models are applied to the image patches in the training dataset $S$.

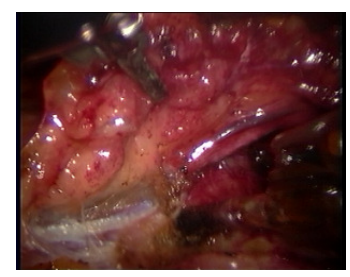

(a)

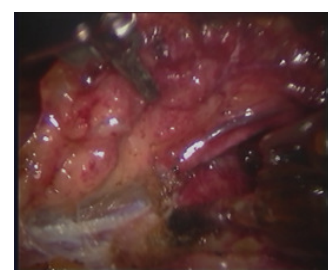

(b)

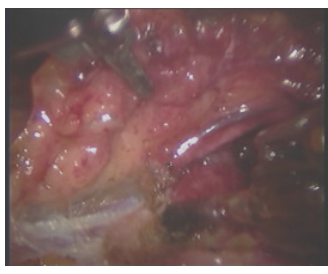

(c)

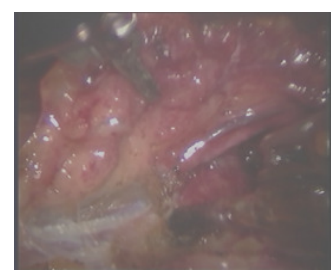

(d)

Fig. 4. Generating training data with appearance-based modelling. The visual effect of surgical smoke is modelled by using Equation 1. (a) original image, (b) $d=0.15$, (c) $d=0.25$, (d) $d=0.4$. 


\subsubsection{Context Specific Descriptors}

For deriving context specific descriptors, the feature descriptor is represented as a classifier. Given an image patch, the classifier performs a number of tests. According to the outcome of the test the patch will be classify as either feature or not a feature. The classifier employed in this paper is an ID3 (Quinlan, 1986) decision tree and the tests examine the relative pixel values in the image patches. The training of the classifier is discussed in subsequent sections of this paper.

The decision tree classifier is chosen due to its fast execution time. This approach is in keeping with (Lepetit et al., 2005) which demonstrates sets of image patches can be efficiently classified using classically constructed top-down decision trees. The concept of representing features as decision trees is illustrated in Figure 5(a). Each non terminal node in the decision tree contains a test. The test examines the relative values of two pixels at location $\mathrm{P}_{1}$ and $\mathrm{P}_{2}$ in the image patch and classifies it such that

$$
\begin{aligned}
& \text { if }\left(\mathrm{P}_{1}>\mathrm{P}_{2}\right) \quad \text { Classify as child node } 1 \\
& \operatorname{if}\left(\mathrm{P}_{1} \approx \mathrm{P}_{2}\right) \quad \text { Classify as child node } 2 \\
& \operatorname{if}\left(\mathrm{P}_{1}<\mathrm{P}_{2}\right) \quad \text { Classify as child node } 3
\end{aligned}
$$

Each leaf node in the tree contains a label and a distribution. The label is either feature or not a feature. The distribution is determined by the number of image patches in the training set $S$ that have been classified by said leaf node.

\subsubsection{Feature Tracking with Context Specific Descriptors}

Tracking a feature in a new video frame is achieved by classifying image patches taken from the new frame. The image patches are dropped down the decision tree and classified as feature or not a feature. The feature's location in the new image is the image patch that is classified as a feature. In practice, however, it is likely that more than one image patch will be classified as a feature. Patches classified as a feature are usually clustered within a few pixels of the true position of the feature. This is because the image patches around the true location of the feature may be visually similar only with a small offset. The training data contains examples of the feature undergoing image transformations and therefore it is likely to classify image patches with a small offset as a feature.

The feature is localised by examining the probability distribution $P\left(N_{j}\right)=|a||b|^{-1}$ at the tree leaf node $N_{j}$ to determine if it is the best match. $|a|$ is the number of feature image patches classified by node $j .|b|$ is the number of feature image patches classified by the entire tree. 
This probability is combined with a spatial weighting to ensure temporal persistency of the tracking. The weighting is a Gaussian kernel centred at the last known position of the feature. The feature's position in the new frame is localised as the image patch with the largest weighted probability.

\subsubsection{Context Specific Descriptors Update}

If the feature is successfully tracked in the image using the context specific descriptor an image patch centred on the tracked position is extracted, labelled as feature and added to the decision tree. This alters the distribution of the data in the decision tree. It enables small changes in the feature descriptor to be accounted for over time without discarding the information used to learn the context specific descriptor. At each frame, patches are randomly chosen from the image, labelled as not a feature and added to the decision tree. If these image patches are incorrectly classified, the classifier is retrained.

\subsection{Learning Context Specific Descriptors}

An overview of the framework for learning a context specific descriptor is provided in Figure 2. As stated above, the descriptor is represented as a decision tree classifier. The role of the classifier is to categorize image patches as a feature or not a feature according to the outcome of a number of tests. In this section, we will describe how to efficiently select the optimal tests and how the classifier can be iteratively evaluated and updated to learn a context specific feature descriptor.

\subsubsection{Training the Classifier}

Using the methods described above, a set of training data $S$ is generated. The set $S$ contains two subsets; $S_{t}$ containing all image patches labelled feature and $S_{f}$ containing all image patches labelled not a feature. The ID3 decision tree is initialised with a root node. A test is selected at this node which partitions the data $S$ into three subsets, according to Equation 2. For each subset, another test is selected which partitions each subset into three further subsets. This process continues until the subset is empty or contains only data labelled as feature or data labelled as not a feature. This point in the tree is a leaf or terminal node. Two decision trees are illustrated in Figure 5(a).

The node classification tests examine the relative value of a pair of pixels according to Equation 2. A selection criterion is required to determine which pair of pixels to compare. Figure 5(b) illustrates two tests. The first test, illustrating poor seperability, compares pixels $\mathrm{P}_{1}$ and $\mathrm{P}_{2}$. The second test compares $\mathrm{P}_{3}$ and $\mathrm{P}_{4}$ and has good seperability. The selection criterion function identifies the optimal test or pair of pixels with the highest seperability. 
This function should provide the maximum information allowing the entropy of each subset to be measured such that;

$$
H(S)=|S| \log _{2}|S|-\left|S_{t}\right| \log _{2}\left|S_{t}\right|-\left|S_{f}\right| \log _{2}\left|S_{f}\right|
$$

The optimal test has zero or minimum entropy.

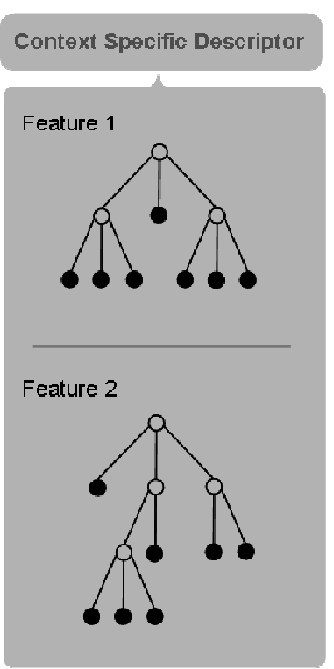

(a)

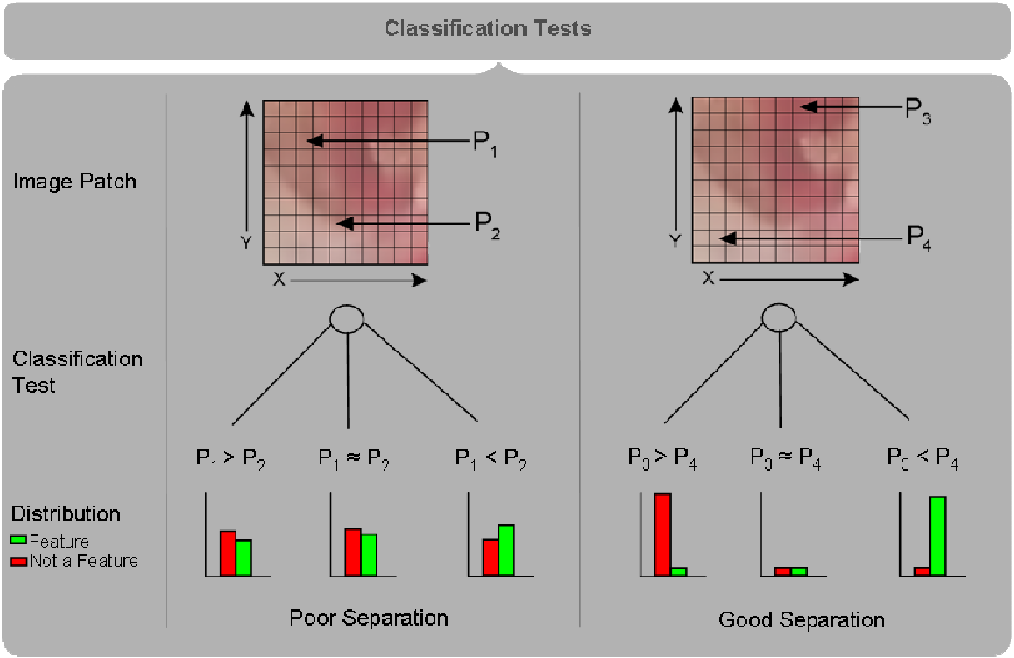

(b)

Fig. 5. The main steps involved in learning context specific descriptors; (a) illustrates the use of ID3 decision trees to represent a feature descriptor. Leaf nodes are shown as a solid black dot and non terminal nodes are shown as circles with black edges. Each non terminal node has a classification test associated to it. (b) Shows two classification tests for different pairs of pixels $\left(\mathrm{P}_{1}, \mathrm{P}_{2}\right)$ and $\left(\mathrm{P}_{3}, \mathrm{P}_{4}\right)$. The examples shown above indicates that $\left(\mathrm{P}_{1}, \mathrm{P}_{2}\right)$ has poor separation and $\left(\mathrm{P}_{3}, \mathrm{P}_{4}\right)$ has good separation.

The optimal test can be found by using an exhaustive search strategy: this can be computationally prohibitive for large datasets. Given a patch of size $w=x^{*} y$, and a set of patches $S$ of size $k$, an exhaustive search requires up to $(w-1) * w / 2 * k$ operations. Computational performance can be improved by sub-sampling $w$ and $k$, however, this can adversely affect the quality of the descriptor. Instead, a search step is introduced without the $w / 2$ component, which examines the distribution of pixels at individual locations. This step searches for individual pixel locations (e.g. $\mathrm{P}_{3}$ in Figure 5) that yield a good separation between the sets $S_{t}$ and $S_{f}$. This is achieved by examining the distribution of pixel values, at a given location, for all image patches in $S$. These pixels are more likely to be part of a pair of pixels that provide optimal partitions. A selection criterion is required to identify intra- and inter-class variance. Linear discriminant analysis may be used if the distribution of the set is uni-modal, as illustrated in Figure 6(a). However, as shown in Figure 6(c), the distribution can be multimodal. It is shown in (Collins et al., 2005) that the log likelihood ratio is wellsuited to the evaluation of multimodal distributions. At each pixel location, two histograms 
are created, $t(x, y)$ and $f(x, y)$, which correspond to $S_{t}$ and $S_{f}$ of the currently evaluated set. The histograms contain the distribution of the pixel values. The log likelihood is computed

$$
L(x, y)=\log \frac{\max (t(x, y), \delta)}{\max (f(x, y), \delta)}
$$

where $\delta$ is set to be 0.001 to avoiding dividing by zero. The variance ratio of the log likelihood is used to measure the intra- and inter-class variance, i.e.

$$
V(L ; x, y)=\frac{\operatorname{var}(L ;(t+f) / 2)}{[\operatorname{var}(L ; t)+\operatorname{var}(L ; f)]}
$$

where

$$
\operatorname{var}(L ;(a))=\sum_{i} a(i) L^{2}(i)-\left(\sum_{i} a(i) L(i)\right)^{2}
$$

given the discrete probability density function $a_{i}$. This selection criterion function rewards low intra-class variance and high inter-class variance and identifies pixels with good separability. Individual pixels are ranked according to their log likelihood and pixel pairs for test are selected according to the ranking. This increases the likelihood of finding an optimal test more efficiently using Equation 3.

The final step is the selection of the most discriminative color space for tracking. This follows the criterion set out in (Collins et al., 2005) where forty-nine color spaces are searched in order to identify the most discriminative. This is a linear combination of Red, Green and Blue (RGB) defined as

$$
F_{1}=\left\{w_{1} R+w_{2} G+w_{3} B \mid w_{*} \in[-2,-1,0,1,2]\right\}
$$

thus creating a set of color spaces including RGB, intensity, approximate chrominance, and excess color. The most discriminative color space is identified using the variance ratio outlined in Equation 4.

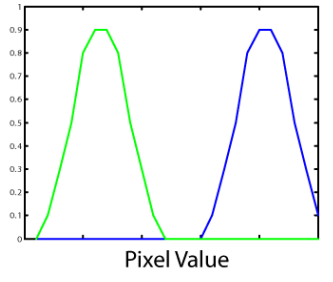

(a)

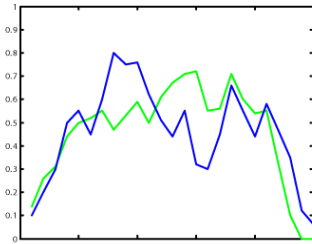

Pixel Value

(b)

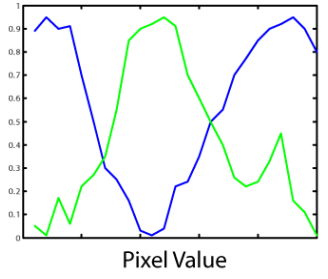

(c)

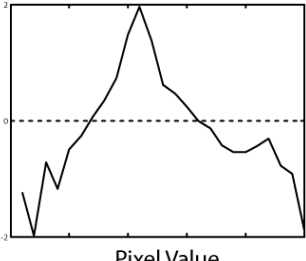

Pixel Value

(d)

Fig. 6. Example distributions of sets $S_{t}$ (green) and $S_{f}$ (blue). (a) Uni-modal distributions with low intra-class variance and high inter-class variance, (b) distributions with high intra-class variance and high inter-class variance, (c) multimodal distributions with low inter-class variance and (d) the log likelihood ratio of multimodal distribution (c). 


\subsubsection{Evaluating and Updating the Classifier}

The performance of the decision tree can be evaluated at any point in time by classifying image patches from the current video frame. The measure of performance is the number of false correspondences. A high number of false correspondences indicate the decision tree is incorrectly classifying the image patches. The performance of the classifier can be improved by adding these image patches to the set of training data $S$ and retraining the classifier. The entire tree does not require retraining; only the leaf nodes responsible for the incorrect classification are retrained. Based on the new distribution of the data, a more informative test is selected.

Computationally, evaluating the classifier is faster than building the decision tree with a large set of training data. This fast evaluation is exploited to reduce build time and optimise performance. The decision tree is built incrementally iterating between updating the decision tree and evaluating its performance. The decision tree is initially built with a small set of training data $S$ and evaluated on the current video frame. It is likely to perform poorly on this data and incorrectly classifies image patches. To resolve this, incorrectly classified patches are added to $S$ and the tree is retrained as described above. This process is repeated until the classifier can correctly classify the feature being tracked. Incrementally building the decision trees online is an efficient and effective approach to creating a context specific classifier and is fundamental to the classifier's performance.

\section{Experiments and Results}

The performance of the proposed approach under scale change, orientation change, surgical smoke, occlusion and deformation resulting from the respiratory and cardiac cycles has been evaluated. It is assessed on simulated and in vivo MIS datasets of the liver, heart and abdomen. The approach is compared to four conventional tracking techniques SIFT (Lowe, 2004), Lucas Kanade (LK) (Lucas and Kanade, 1981) (with template update) and two meanshift algorithms (MS1 and MS2) (Collins et al., 2005).

SIFT detects scale invariant Different of Gaussian (DoG) features and represent greyscale patches around the region as a histogram of oriented gradients. It is a tracking-by-detection approach using nearest neighbour ratio matching (ratio $=0.6$ ) and no temporal information between frames. This makes it well-suited to dealing with occlusion; however, it requires the feature to be redetected in each frame and for said feature to be globally unique in the image. In rigid scenes, matching can be improved by using global matching constraints such as 
Random Sampling Consensus (RANSAC), however, these techniques cannot be easily applied in unknown, non-rigid environments.

LK is a pyramid optical flow method for region tracking. LK uses greyscale spatial information in a template and iteratively attempts to minimise the difference between the template and the observed data. It is based on three key assumptions (Bradski and Kaehler, 2008): 1) brightness constancy, 2) temporal persistence and 3) spatial coherence. LK tracking can suffer from the aperture problem and drift due to template updating required for the temporal persistence criterion.

Mean-shift is a non-parametric, statistically robust method for locating local maxima in a probability density distribution. Mean-shift represents the colour values of the pixels in the patch around the region as a histogram and makes no use of structural information, thus making it capable of tracking deformation. Mean-shift is well-suited to large self-contained blobs and is less suited to lines or corner-like structures. It is assumed that the displacement of a region is small and a spatial overlap of the feature exists between frames. Mean-shift is theoretically robust to partial occlusion but not full occlusion. In this work two mean-shift approaches outlined in (Collins et al., 2005) are used. These mean-shift trackers are more discriminative than the standard approach and attempt to find the optimal colour representation of the region.

To evaluate the performance of the tracking methods the evaluation criteria outlined in (Ke and Sukthankar, 2004) and (Mikolajczyk and Schmid, 2005) are used. Two performance metrics are computed. The sensitivity (also known as recall) is computed as sensitivity $=\frac{\# \text { correct matches }}{\# \text { correspondences }}$ where the number of correspondences is the number of features which exist in the current image and are trackable. A good feature tracking method will have high sensitivity as this is a measure of the density of features. The second performance metric is $\Lambda=1-$ precision. This is a measure of the total number of incorrectly tracked features with respect to the total number of tracked features such that $\Lambda=1-$ precision $=\frac{\# \text { incorrect matches }}{\# \text { correct matches }+\# \text { incorrect matches }}$. A good feature tracker will have a low $\Lambda$ value. This provides an evaluation of the temporal persistency of the feature trackers. 


\subsection{Simulated Experiment with Known Ground Truth}

To quantitatively evaluate the performance of the proposed method with respect to deformation, a simulation was performed to generate synthetic data. A laparoscopic image of the heart was textured onto a 3D surface as shown in Figure 7(c). The surface was periodically deformed with a mixture of Gaussians simulating cardiac and respiratory tissue deformation. The mesh was deformed for 4000 frames. For each frame, the simulation produced an image and the ground truth position of 100 features for quantitative analysis. Cardiac, respiratory motion and noise are simulated however, specular reflection and changing lighting conditions are not.

In Figure 7(b), the trackers are quantitatively evaluated with respect to sensitivity over time. It can clearly be seen that there is oscillation in the performance of all the trackers. This oscillation corresponds to the periodic nature of the applied deformation. The reduction in performance occurs at the extremes of deformation. At these extremes, the regions have changed shape, and localising the centre of the region accurately may fail given that the region tracking methods are drawn towards prominent information in the patch (such as edges or corners which may not be at the centre of the patch).

The oscillation effect is particularly noticeable with SIFT. The change in shape of the region makes accurate repeatability of the DoG detector challenging and violates the geometric constraints imposed by the SIFT descriptor. As deformation of the surface increases, the number of regions, which may accurately be matched using a histogram of gradients, is reduced. This oscillation makes SIFT less attractive for continuous tracking in MIS. Figure 7(d) shows SIFT has the lowest $\Lambda$. This is a result of the matching strategy. The nearest neighbour ratio test prevents a feature from matching if it is visually similar to another detected feature. This prevents false matches.

The LK tracker performs well at the beginning of the experiment with high sensitivity and low values of $\Lambda$. The temporal persistence and spatial coherence assumptions are held in the simulated data. The noise added to the system violates the brightness constancy assumption. Combined with non-linear deformation, the noise causes error propagation when the template is updated: the tracker's performance degrades over time.

The two mean-shift trackers perform similarly. The algorithms have low sensitivity and are only able to track a small number of features accurately. This poor performance can be attributed to three factors: 1) for the majority of detected features, color in MIS images is not sufficient to distinguish the feature from its surroundings. This results in a high value of $\Lambda$ 
and a high number of wrongly matched features; 2) the feature detectors extract edges, corners and blobs in greyscale images, however, mean-shift works well with large selfcontained regions of distinct color. 3) mean-shift assumes a spatial overlap between frames. Small or non existing overlap causes the pixels to fall outside the basin of attraction and leads to tracking failure.

The proposed context specific descriptor maintains a good performance in the presence of deformation with a derived sensitivity outperforming the alternative approaches. The corresponding $\Lambda$ value is low and only outperformed by SIFT. The robustness to synthetic deformation can be attributed to learning from example data. By learning from example data generated by the recursive tracker, the classifier is built on real image transformations, which will be subsequently observed due to the periodic nature of the deformation. Although the context specific descriptor performs well, there remain points that cannot be tracked, and there is fluctuation in performance. This is attributable to noise in the image or poor initialisation from the LK tracking.
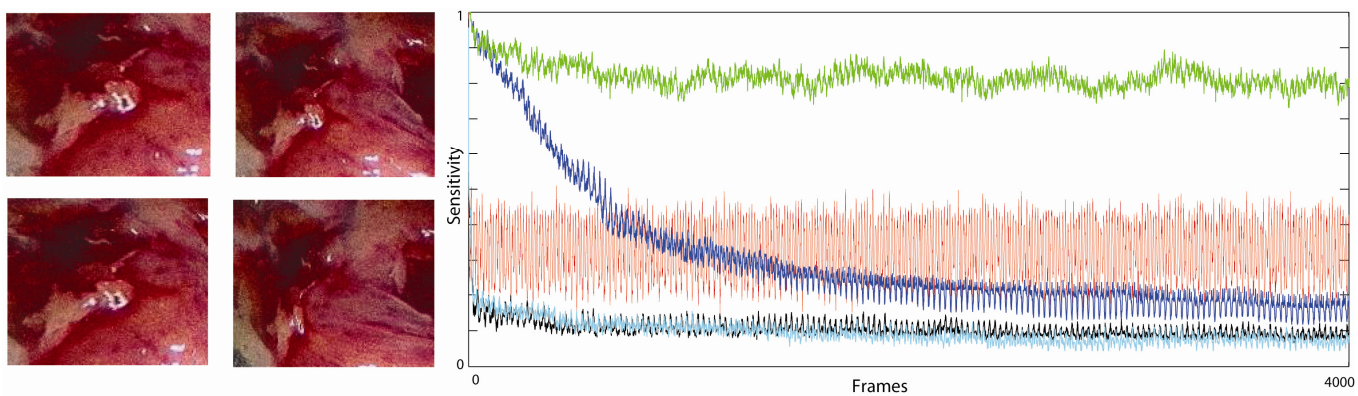

(a)

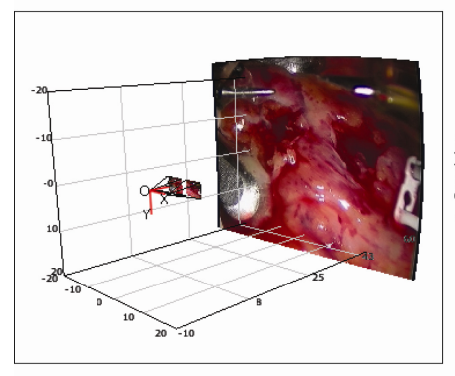

(c)

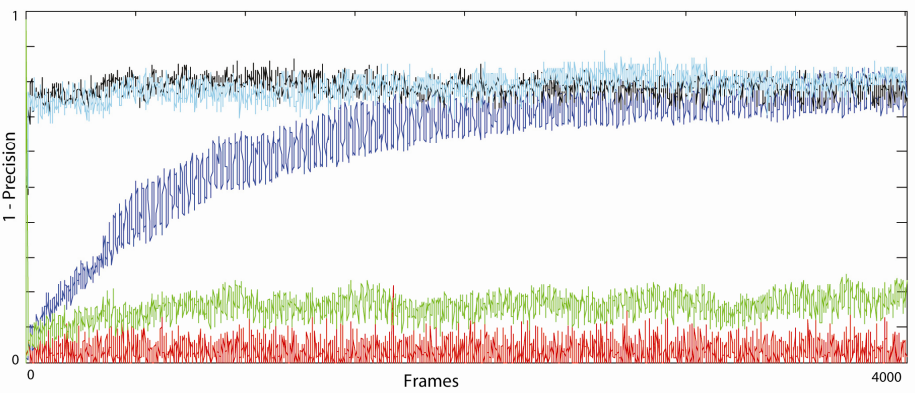

(d)

Fig. 6. Relative tracking performance on synthetic data for the five tracking algorithms considered. (a) The simulated data is created by warping an image taken from a MIS procedure with known ground truth deformation characteristics. (c) Simulation framework. (b) and (d) Relative performance values for the five different tracking techniques compared; green - context specific descriptor, red - SIFT, dark blue - Lucas Kanade, black - meanshift 1 and light blue - mean-shift 2. 


\subsection{In vivo Experiments}

The performance of the proposed technique was quantitatively evaluated on in vivo data. A total of 50 features were detected in the first frame of each sequence. An expert user manually obtained ground truth data for each feature in these sequences at 50 frame intervals.

\section{Tissue deformation}

Figure 8 and Table 1 show the three sequences used and the corresponding tracking results. Figures 8(a-f) show sequences taken from two Totally Endoscopic Coronary Artery Bypass graft (TECAB) cases. Centred in the endoscopic image is the epicardial surface deforming with cardiac and respiratory motion. Rapid tissue deformation and specular reflections make tracking challenging in these sequences.

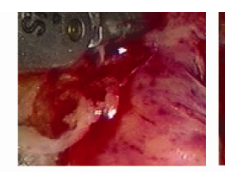

(a)

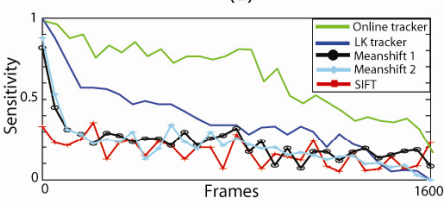

(b)

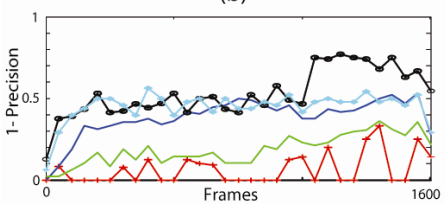

(c)

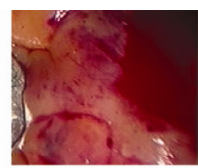

(d)

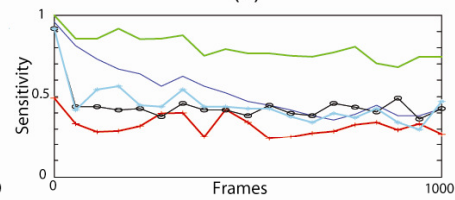

(e)

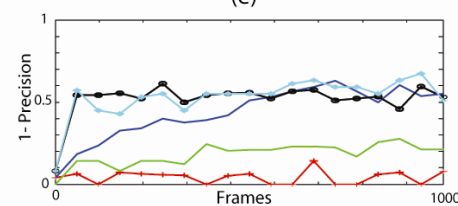

(f)

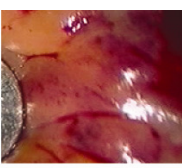

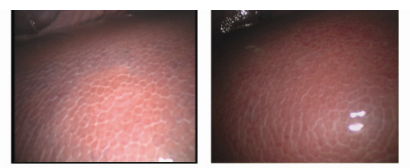

(g)

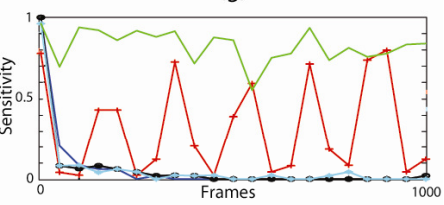

(h)

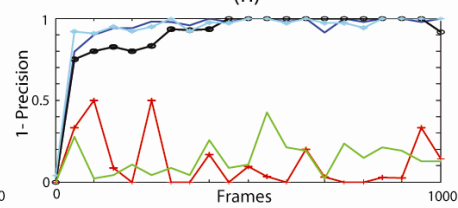

Fig. 8. Relative tracking performance for in vivo deformation sequences. (a-c) In vivo cardiac dataset and tracking analysis. (d-f) Second in vivo cardiac dataset and tracking analysis. (g-i) Porcine in vivo liver dataset and tracking analysis,. Five trackers are compared; green - context specific descriptor, red - SIFT, dark blue - Lucas Kanade, black - mean-shift 1 and light blue - mean-shift 2 .

The relative performance of the trackers on in vivo data is similar to that on synthetic data. In TECAB sequences of Figures 8(a) and 8(d), the LK tracker performs well at the start; however the performance degrades over time as drift occurs. Brightness constancy is violated here due to specular reflections and the change in visual appearance as a result of the point light source. The mean-shift trackers performed worse in the first sequence shown in Figure 8(a) than the second sequence shown in Figure 8(d). This is because deformation in the first sequence is more pronounced leading to larger inter frame motions. SIFT performs poorly in this sequence because larger deformation leads to larger changes in visual appearance. The SIFT descriptor is affected by the specular highlights which cause sharp gradients. Feature density and persistency are higher using the context specific descriptor as shown in Figure 8(b) and 8(e) and surmised in Table 1. 
Table 1. Sensitivity and $\Lambda$ for different techniques compared for tissue deformation tracking.

\begin{tabular}{cccccc}
\hline & LK & MS1 & MS2 & SIFT & $\begin{array}{c}\text { Context Specific } \\
\text { Descriptor }\end{array}$ \\
\hline Sensitivity & 0.333 & 0.245 & 0.241 & 0.287 & 0.752 \\
$\Lambda$ & 0.555 & 0.545 & 0.586 & 0.049 & 0.170 \\
\hline
\end{tabular}

Figure $8(\mathrm{~g})$ shows footage of the liver deforming as a result of respiration. Deformation is less pronounced than on the cardiac surface; however, illumination of the surface changes significantly as the organ moves toward, and away, from the laparoscope and light source. The LK tracker performs poorly in this footage. This is attributed to changes in scale and illumination, which violate the brightness constancy assumption. The cyclical performance of SIFT is seen again in this sequence. The DoG detector has relatively low repeatability on this sequence because the structures on the liver are small and have low contrast. The mean-shift algorithms do not perform well due to the uniform colour distribution of the liver. The context specific descriptor performs well on this sequence not only because it is learning the most discriminative information but also because the representation of the information is robust to changes in illumination.

\section{Occlusion}

The use of surgical tools during MIS leads to occlusion of the operative field and full or partial occlusion of features on the surface of the tissue. Figures $9(\mathrm{a}, \mathrm{d}, \mathrm{g})$ show deformation of the liver and abdomen wall resulting from respiration. The surgeon introduces tools in these sequences leading to occlusion. Quantitative analysis is provided in Figure 9 and Table 2. In Figure 9(a), only a small number of regions are occluded starting at frame 200. At this point, the decreased performance of the LK and mean-shift trackers is clear. These trackers require temporal information and have no explicit mechanism for dealing with recovery from full occlusion. SIFT and the context specific descriptor remain robust in the presence of occlusion. In Figure 9(d), the number of occluded regions increases. The trackers' performances are similar to that of the first sequence. The context specific descriptor outperforms SIFT on these sequences; however, both have a low $\Lambda$ value, thus demonstrating the correct identification of occluded regions. 

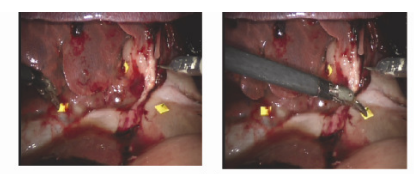

(a)

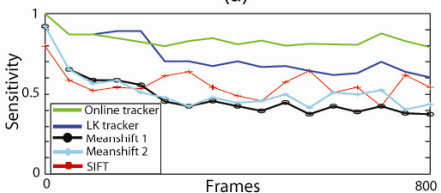

(b)

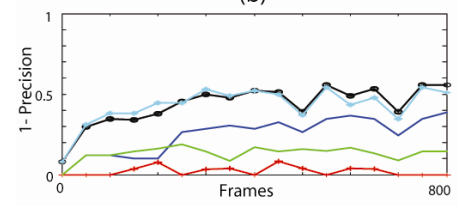

(c)
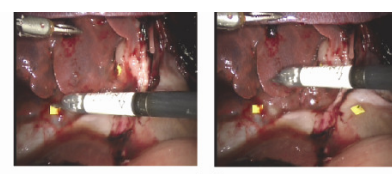

(d)

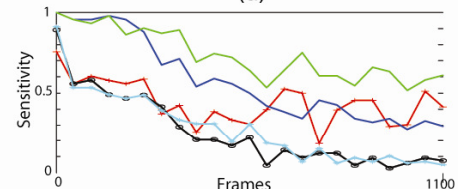

(e)

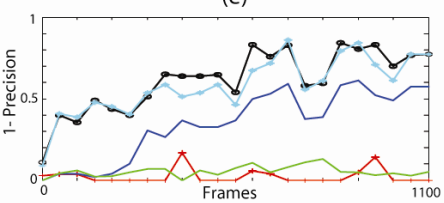

(f)

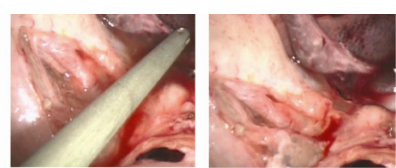

(g)

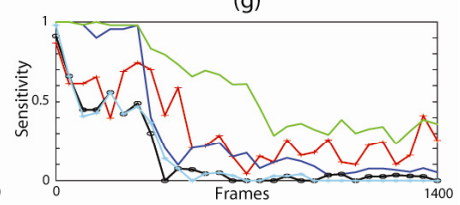

(h)

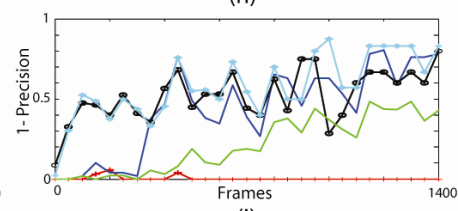

(i)

Fig. 9. Relative tracking performance for in vivo occlusion sequences. (a-c) Occlusion sequence one with tracking analysis. (d-f) Occlusion sequence two with tracking analysis. (g-i) Occlusion sequence three with tracking analysis. Five trackers are compared; green - context specific descriptor, red - SIFT, dark blue - Lucas Kanade, black - mean-shift 1 and light blue - mean-shift 2 .

In the last sequence shown in Figure $9(\mathrm{~g})$, the surgeon uses an irrigation tool. This tool occludes the majority of the regions after 300 frames. At this point, there is a significant reduction in the performance of LK and mean-shift (almost zero sensitivity). At around frame 700 , the suction tool interacts with tissue to remove blood from the surface. This leads to a reduction in sensitivity of the context specific descriptor and causes an increase in the value of $\Lambda$. This is attributed to the change in visual appearance of the scene due to the removal of blood. This scene is different to the data used to train the classifier.

Table 2. Sensitivity and $\Lambda$ corresponding to different feature trackers when tissue occlusion is encountered.

\begin{tabular}{cccccc}
\hline & LK & MS1 & MS2 & SIFT & $\begin{array}{c}\text { Context Specific } \\
\text { Descriptor }\end{array}$ \\
\hline Sensitivity & 0.557 & 0.307 & 0.322 & 0.458 & 0.728 \\
$\Lambda$ & 0.356 & 0.500 & 0.494 & 0.017 & 0.144 \\
\hline
\end{tabular}

\section{Scale and Rotation}

In MIS, the surgeon frequently manipulates the laparoscope to explore and localise the target anatomy. This leads to changes in scale and rotation of the images. Figure $10(\mathrm{~g})$ illustrates the effect of camera motion. The laparoscope is navigated towards and away from the abdomen wall causing a scale change factor of between 2.8 and 0.66 . As a result of the significant scale change only 18 features detected in the first frame are visible throughout the entire sequence. 
The performance of the trackers with respect to changes in scale is quantitatively evaluated in Figures 10(h-i). The LK tracker does not explicitly incorporate scale information; however, it is able to track the features due to the template update and temporal persistence assumption. The sensitivity reduces towards the end of the sequence as a result of error propagation and drift. The mean-shift trackers are generally not invariant to scale changes although the approach has been extended in (Bradski, 1998) for scale. The high value of $\Lambda$ demonstrates again that color alone is not sufficient for dense feature tracking. SIFT detects multi-scale DoG features which are theoretically robust to changes in scale. However, the sensitivity of SIFT decreases as scale changes and the number of wrongly matched features significantly increases when the scale is reduced to 0.66 . The proposed context specific descriptor explicitly incorporates scale into the learning process enabling it to remain robust to changes in scale and out perform the conventional trackers.
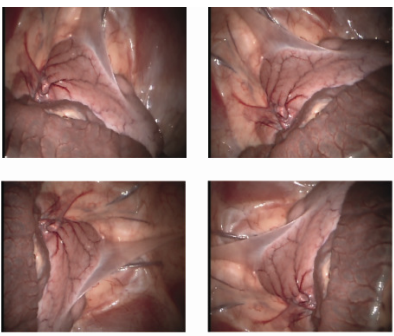

(a)

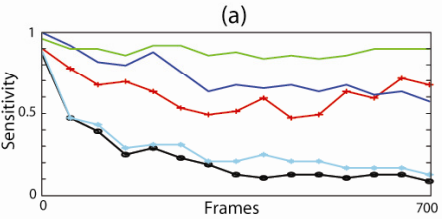

(b)

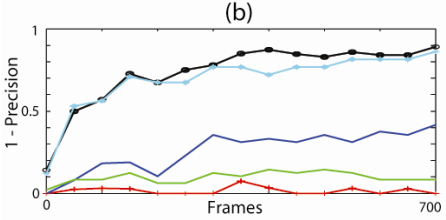

(c)
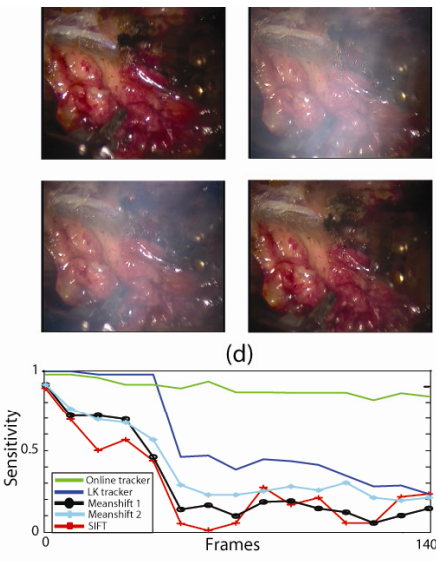

(e)

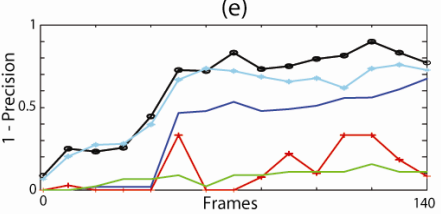

(f)

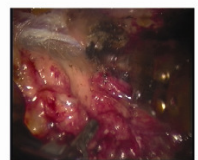

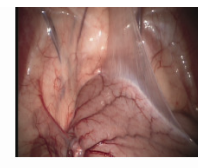
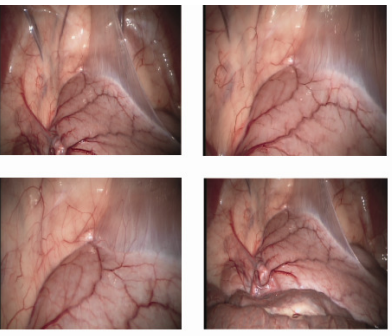

(g)

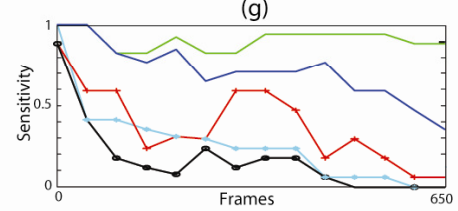

(h)

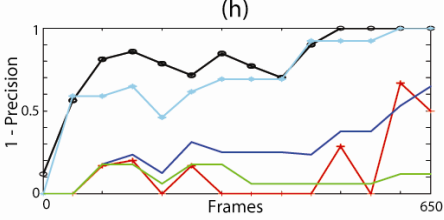

(i)

Fig. 10. Relative tracking performance for in vivo sequences with respect to rotation, surgical smoke and scale change. (a-c) Rotation around the optical axis with tracking analysis. (d-f) Surgical smoke resulting from diathermy with tracking analysis. (g-i) Scale change with tracking analysis. Five trackers are compared; green context specific descriptor, red - SIFT, dark blue - Lucas Kanade, black - mean-shift 1 and light blue - meanshift 2.

With a rigid laparoscope, the movement of the tip of the scope is limited by the fulcrum effect at the trocar port (Crothers et al., 1999). As a result, the most commonly observed rotation is around the optical axis as shown in Figure 10(a). In Figures 10(b) and 10(c) the effect of rotation is quantitatively evaluated on footage of the abdomen. The laparoscope is first rotated approximately $50^{\circ}$ anticlockwise, it is then returned to its original position and rotated $120^{\circ}$ clockwise. The LK tracker and mean-shift trackers do not explicitly represent information 
about orientation. However, the LK tracker is able to perform well in this sequence due to template update. The inter-frame motion of the camera is small which means the temporal persistence assumption is held. The mean-shift trackers do not represent spatial information and are theoretically invariant to rotation, the poor performance here can be attributed to the lack of distinct color variation. SIFT, which computes the orientation of the DoG features prior to matching, is shown to be robust to changes in orientation; however, feature density is higher with the context specific descriptor which performs consistently well under the observed rotations.

\section{Surgical Smoke}

In Figures 10(d-f), the effect of smoke resulting from diathermy is investigated. In this sequence, the diathermy is activated twice causing smoke shown in Figure 10(d). The build up of smoke is gradual over a number of frames and remains visible for a short period before a suction device is used to remove it. Translucent smoke has the effect of greying the pixels, reducing the colour distribution; flattening gradients and making the image appear more homogenous.

This affects the SIFT features as the histogram of gradients will be changed making matching harder. It can be seen in Figure 10(e) that when the diathermy is activated causing smoke, the sensitivity for SIFT drops to zero. The gradual flattening of gradients during the appearance of smoke means the LK tracker has less structure on which to converge. This results in template drift as the smoke is incorporated into the template. On this sequence, the mean-shift tracker performs well at the beginning with high sensitivity. This is attributed to a number of self contained blobs of distinctive colour on the surface of the tissue. However, the greying of the pixels changes the colour distribution of the feature patches. As a result, the model of colour distribution for the mean-shift trackers is no longer valid. The context specific descriptor performed well because the greying of the pixels and flattening of gradients has been simulated and incorporated into the training data. Our approach has therefore learnt what information in the scene can be used for tracking in the presence of smoke. The context specific descriptor remains robust to smoke; however, there is a slight drop in performance which is attributed to the smoke modelling which does not accurately capture the non uniform spatial distribution.

Table 3. Sensitivity and $\Lambda$ for scale, rotation and surgical smoke.

\begin{tabular}{cccccc}
\hline & LK & MS1 & MS2 & SIFT & $\begin{array}{c}\text { Context Specific } \\
\text { Descriptor }\end{array}$ \\
\hline Sensitivity & 0.665 & 0.263 & 0.332 & 0.453 & 0.892 \\
$\Lambda$ & 0.278 & 0.606 & 0.571 & 0.032 & 0.076 \\
\hline
\end{tabular}




\subsection{Computational Performance Analysis}

The proposed framework was implemented on a desktop PC with an Intel Pentium $3 \mathrm{GHz}$ processor and 2GB of RAM. The software was written in C++ on a Microsoft Windows environment. The most computationally demanding component of the framework is learning the context specific descriptor. In Figure 11, the average computational requirements for learning a single feature is shown. The figure compares computation timings for exhaustive search and the optimised search in Figures 11(a) and 11(b) respectively. The optimised search is approximately 10 times faster.

The graphs in Figure 11 share a similar profile. The computation cost is initially high; this corresponds to building the initial classifier. Subsequent peaks in the graph are the result of retraining the classifier and adaptively updating it. After 20 frames, the requirement to retrain the classifier is reduced. This implies that a context specific descriptor has been learnt and it is well-suited to the current endoscopic images. If the images significantly change or a new object is introduced into the scene the classifier may retrain to take this into account. Tracking the feature with the context specific descriptor is computationally efficient. The average time required to track a feature is $1.9 \mathrm{~ms}$ with a standard deviation of $0.62 \mathrm{~ms}$. As discussed earlier, feature tracking and learning the context specific descriptor are independent processes which can be performed in parallel to avoid computational bottlenecks.

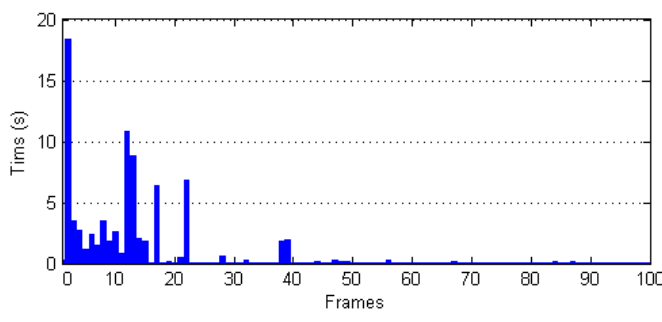

(a)

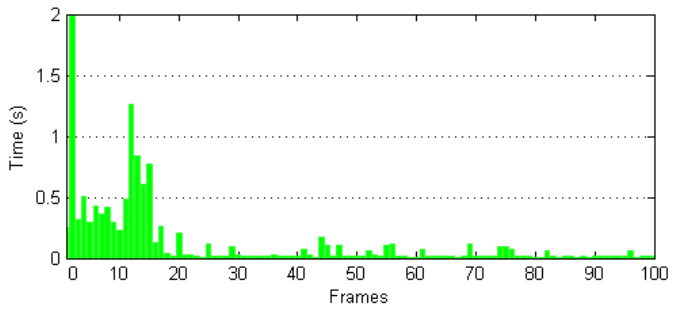

(b)

Fig. 11. The computational requirements of the learning phase of the system shown for (a) exhaustive search and (b) optimised search.

\section{Discussions}

In this study, it has been shown that the LK tracker had high feature density and persistency in the presence of deformation, scale change and rotation where the algorithm's assumptions are held; however, by performing template update the approach eventually succumbs to error propagation and drift. Tracking without the template update restricts how much the feature 
patch is allowed to change and as a result, restricts tracking capabilities. The LK approach was found to be sensitive to illumination changes and has no explicit mechanism for dealing with occlusion. Robustness to occlusion and drift can be improved by incorporating feature redetection as proposed in (Richa et al., 2010). The window size was set constant for all video sequences. Performance could be improved by empirically adjusting the window size for individual sequences as shown in (Masson et al., 2009).

The results show that the mean-shift trackers are not well-suited to tracking most of the features detected on the surface of tissue leading to low feature density. In these video sequences, colour alone is not a unique enough characteristic for tracking features. Feature tracking may also fail under rapid motion where the feature falls outside the basin of attraction. However, it should be noted that when mean-shift can track a feature it is robust to image transformations including deformation, rotation and partial occlusion. Both algorithms performed at a similar level with the second approach performing slightly better. Alternative feature detectors that extract colour features such as maximally stable colour regions (Forss, 2007) may help improve results.

SIFT is not designed to be used as a tracker but rather for wide baseline matching. This tracking-by-detection approach means it recovers well from occlusion and tracking failure; however, the histogram of gradients used to represent the feature does not deal well with tissue deformation or specular highlights. The approach can fail if the repeatability of the detector is low. With tracking-by-detection strategies there is a trade off between detecting too many points, which makes matching more challenging and is computationally expensive, and not detecting enough points leading to low repeatability and tracking failure. This is a compromise between feature density and persistency. The DoG detector is not invariant to deformation and therefore may not accurately localise the centre of detected points consistently under observed deformation. This is because the DoG identifies what structural information is important and uses this to guide the localisation. Matching results may be improved with a more sophisticated matching strategy incorporating temporal information, prior knowledge or global outlier removal. However, if the gradient histogram is not invariant to the types of deformation and image transformations observed in MIS the improved matching strategy will have limited effect. Relaxing the spatial constraints of the gradient histogram may increase invariance to deformation but is likely to make the descriptors less discriminative.

The results presented in this paper show that the context specific descriptor performed consistently well under all image transformations with good feature density and persistency. 
Good density and persistency can both be attributed to the learning approach. Feature density is high because the approach learns what is unique about a feature relative to its surroundings. Feature persistency is high because the descriptor learns directly from the data what types of image transformation will be experienced and what information is robust to these image transformations. This approach is particularly well-suited to tracking periodically deforming tissue; however, its robustness is limited by the set of training data used to build the classifier.

\section{Conclusions}

In this paper, we have provided a detailed explanation of context specific descriptors for tracking deforming tissue in MIS. By extending our previous work (Mountney and Yang, 2008), the method generates and automatically labels context specific data. The data is used to train a classifier and learn the information which discriminates a feature from its surroundings. The proposed method is made robust to large scale image transformations by synthetically generating data with geometric and appearance models. To improve the computational performance of the training process, we have included an additional search step based on log likelihoods and demonstrated how the classifier can be improved by online evaluation and adaptive updates. The approach has been validated on simulated and in vivo data. Its performance has been compared to four conventional tracking techniques. We have demonstrated that the technique is robust to drift, capable of recovering from occlusion, robust to changes in scale and orientation and the presence of diathermy induced smoke. Future work will focus on using multiple decision trees to improve feature representation, cotraining to improve performance, parallelisation and the development of more sophisticated appearance modelling.

\section{Acknowledgement}

The research presented in this paper is supported by the EU PASSPORT project and an UK EPSRC/TSB (Technology Strategy Board) grant (DT/E011101/1). The authors would like to thank Danail Stoyanov for his help and advice.

\section{Authors}

Peter Mountney (peter.mountney05@imperial.ac.uk) received his PhD in Medical Imaging from Imperial College London in 2010. He is currently working as a Research Associate at Imperial College. His research interests are in the use of laparoscopic cameras as a means of 
providing measurement of tissue structure during MIS. His current work focuses on imageguided surgery, robot navigation, soft tissue tracking, 3-D estimation, tissue modelling and SLAM.

\section{References}

- Albitar, C., Graebling, P., Doignon, C., 2007. Robust Structured Light Coding for 3D Reconstruction, International Conference on Computer Vision, pp. 1-6.

- Amit, Y., Geman, D., 1997. Shape Quantization and Recognition with Randomized Trees, Neural Computation, pp. 1545-1588.

- Bradski, G.R., 1998. Computer video face tracking for use in a perceptual user interface. Intel Technology Journal Q2.

- Bradski, G.R., Kaehler, A., 2008. Learning OpenCV: Computer Vision with the OpenCV Library.

- Collins, R., Liu, Y., Leordeanu, M., 2005. On-Line Selection of Discriminative Tracking Features. IEEE Transactions on Pattern Analysis and Machine Intelligence 10(27), 16311643.

- Comaniciu, D., Ramesh, V., Meer, P., 2003. Kernel-Based Object Tracking. IEEE Transactions on Pattern Analysis and Machine Intelligence 25, 564-577.

- Crothers, I., Gallagher, A., McClure, N., James, D.T.D., McGuigan, J., 1999. Experienced laparoscopic surgeons are automated to the "fulcrum effect": an ergonomic demonstration. Endoscopy 318, 365-369.

- Deligianni, F., Chung, A., Yang, G.-Z., 2003. 2D/3D Registration Using Shape from Shading Information in Application to Endoscope, Medical Image Understanding and Analysis, pp. 33-36.

- Devernay, F., Mourgues, F., Coste-Maniere, E., 2001. Towards endoscopic augmented reality for robotically assisted minimally invasive cardiac surgery, Medical Imaging and Augmented Reality.

- Forss, P.E., 2007. Maximally Stable Colour Regions for Recognition and Matching, Computer Vision and Pattern Recognition, pp. 1-8.

- Fuchs, H., Livingston, M.A., Raskar, R., Colucci, D., Keller, K., State, A., Crawford, J.R., Rademacher, P., Drake, S.H., Meyer, A.A., 1998. Augmented reality visualization for laparoscopic surgery, Medical Image Computing and Computer Assisted Intervention, pp. 934-943.

- Ginhoux, R., Gangloff, J.A., Mathelin, M.d., Soler, L., Sanchez, M.M.A., Marescaux, J., 2004. Beating heart tracking in robotic surgery using $500 \mathrm{~Hz}$ visual servoing, model predictive control and an adaptive observer, International Conference on Robotics and Automation, pp. 274- 279.

- Gröger, M., Ortmaier, T., Sepp, W., Hirzinger, G., 2002. Tracking local motion on the beating heart, SPIE Medical Imaging Conference, San Diego, USA, pp. 233-241.

- Höller, K., Petrunina, M., Penne, J., Schneider, A., Wilhelm, D., Feußner, H., Hornegger, J., 2008. Taking endoscopy to a higher dimension: Computer Aided 3-D NOTES 4th Russian-Bavarian Conference on Biomedical Engineering pp. 33-37.

- Ke, Y., Sukthankar, R., 2004. PCA-SIFT: A More Distinctive Representation for Local Image Descriptors, Computer Vision and Pattern Recognition, pp. 506-513.

- Koppel, D., Chen, C.-I., Wang, Y.-F., Lee, H., Gu, J., Poirson, A., Wolters, R., 2007. Toward automated model building from video in computer-assisted diagnoses in colonoscopy, SPIE. 
- Lau, W.W., Ramey, N.A., Corso, J., Thakor, N.V., Hager, G.D., 2004. Stereo-Based Endoscopic Tracking of Cardiac Surface Deformation, Medical Image Computing and Computer Assisted Intervention, St. Malo, France, pp. 494-501.

- Lepetit, V., Lagger, P., Fua, P., 2005. Randomized trees for real-time keypoint recognition, Computer Vision and Pattern Recognition, pp. 775-781.

- Lowe, D.G., 2004. Distinctive Image Features from Scale-Invariant Keypoints. International Journal of Computer Vision 60, 91-110.

- Lucas, B., Kanade, T., 1981. An Iterative Image Registration Technique with an Application to Stereo Vision, International Joint Conference on Artificial Intelligence, pp. 674-679.

- Masson, N., Nageotte, F., Zanne, P., Mathelin, M.d., 2009. In Vivo Comparison of Realtime Tracking Algorithms for Interventional Flexible Endoscopy, International Symposium on Biomedical Imaging, pp. 1350-1353.

- Mikolajczyk, K., Schmid, C., 2005. A performance evaluation of local descriptors. IEEE Transactions on Pattern Analysis and Machine Intelligence 27, 1615-1630.

- Mountney, P., Lo, B.P.L., Thiemjarus, S., Stoyanov, D., Yang, G.-Z., 2007. A Probabilistic Framework for Tracking Deformable Soft Tissue in Minimally Invasive Surgery, Medical Image Computing and Computer Assisted Intervention, pp. 34-41.

- Mountney, P., Yang, G.-Z., 2008. Soft Tissue Tracking for Minimally Invasive Surgery: Learning Local Deformation Online, Medical Image Computing and Computer Assisted Intervention, pp. 364-372.

- Mylonas, G.P., Stoyanov, D., Deligianni, F., Darzi, A., Yang, G.-Z., 2005. GazeContingent Soft Tissue Deformation Tracking for Minimally Invasive Robotic Surgery, Medical Image Computing and Computer Assisted Intervention, pp. 843-850.

- Noce, A., Triboulet, J., Poignet, P., 2007. Efficient Tracking of the Heart Using Texture, Annual International Conference of the IEEE Engineering in Medicine and Biology Society, pp. $4480-4483$.

- Noce, A., Triboulet, J., Poignet, P., Dombre, E., 2006. Texture Features Selection for Visual Servoing of the Beating Heart, Biomedical Robotics and Biomechatronics, pp. 335 -340 .

- Okatani, T., Deguchi, K., 1997. Shape Reconstruction from an Endoscope Image by Shape from Shading Technique for a Point Light Source at the Projection Centre. Computer Vision and Image Understanding 66, 119-131.

- Ortmaier, T., Gröger, M., Boehm, D.H., Falk, V., Hirzinger, G., 2005. Motion Estimation in Beating Heart Surgery. IEEE Transactions on Biomedical Engineering 52, 1729-1740.

- Ott, L., Zanne, P., Nageotte, F., Mathelin, M.d., Gangloff, J., 2008. Physiological motion rejection in flexible endoscopy using visual servoing, International Conference on Robotics and Automation, pp. 2928-2933.

- Quartucci Forster, C., H,. Tozzi, C,L,. , 2000. Towards 3D reconstruction of endoscope images using shape from shading, Brazilian Symposium on Computer Graphics and Image Processing, pp. 90-96.

- Quinlan, J.R., 1986. Induction of decision trees. Machine Learning 1.

- Richa, R., Bó, A.P., Poignet, P., 2010. Robust 3D Visual Tracking for Robotic-assisted Cardiac Interventions, Medical Image Computing and Computer Assisted Intervention, pp. 267-274.

- Richa, R., Poignet, P., Liu, C., 2008. Deformable motion tracking of the heart surface, International Conference on Intelligent Robots and Systems, pp. 3997-4003.

- Rosten, E., Drummond, T., 2006. Machine learning for high-speed corner detection, European Conference on Computer Vision, pp. 430-443.

- Sauvee, M., Poignet, P., Triboulet, J., Dombre, E., Malis, E., Demaria, R., 2006. 3D Heart Motion Estimation using Endoscopic Monocular Vision System, 6th IFAC Symposium on Modelling and Control in Biomedical Systems. 
- Shi, J., Tomasi, C., 1994. Good features to track, Computer Vision and Pattern Recognition, pp. 593-600.

- Spearman, J., . Tsavellas, G,. Nichols, P,. 2007. Current attitudes and practices towards diathermy smoke. Annals of The Royal College of Surgeons of England 4, 162-165.

- Stoyanov, D., Mylonas, G.P., Deligianni, F., Darzi, A., Yang, G.-Z., 2005. Soft-tissue Motion Tracking and Structure Estimation for Robotic Assisted MIS Procedures, Medical Image Computing and Computer Assisted Intervention, pp. 139-146.

- Stoyanov D., Darzi A., Yang G-Y., 2004. Dense 3D Depth Recovery for Soft Tissue Deformation During Robotically Assisted Laparoscopic Surgery, Medical Image Computing and Computer Assisted Interventions, pp. 48-55.

- Wengert, C., Cattin, P.C., Duff, J.M., Székely, G., 2006. Markerless Endoscopic Registration and Referencing, Medical Image Computing and Computer-Assisted Intervention pp. 816--823. 